ARTICLE

\title{
Fear extinction disruption in a developmental rodent model of schizophrenia correlates with an impairment in basolateral amygdala-medial prefrontal cortex plasticity
}

\author{
Daniela L. Uliana ${ }^{1}$, Leonardo B. M. Resstel ${ }^{1}$ and Anthony A. Grace (iD ${ }^{2}$
}

\begin{abstract}
Schizophrenia patients typically exhibit prominent negative symptoms associated with deficits in extinction recall and decreased ventromedial prefrontal cortex activity (vmPFC, analogous to medial PFC infralimbic segment in rodents). mPFC activity modulates the activity of basolateral amygdala (BLA) and this connectivity is related to extinction. mPFC and BLA activity has been shown to be altered in the methylazoxymethanol acetate (MAM) developmental disruption model of schizophrenia. However, it is unknown if there are alterations in extinction processes in this model. Therefore, we investigated extinction and the role of $\mathrm{mPFC}-\mathrm{BLA}$ balance in MAM rats. Male offspring of pregnant rats treated with Saline or MAM ( $20 \mathrm{mg} / \mathrm{kg}$; i.p.) on gestational day 17 were used in fear conditioning (contextual/tone) and electrophysiological experiments (mPFC-BLA plasticity). No difference was observed in conditioning, extinction, and test sessions in contextual fear conditioning. However, MAM-treated rats demonstrated impairment in extinction learning and recall in tone fear conditioning. Furthermore, high frequency stimulation (HFS) of the BLA decreased spike probability in the MPFC of saline-treated rats but not in MAM rats. NMDA antagonist microinjected into the BLA disrupted extinction learning and recall in control rats, resulting in a similar deficit as that observed in MAM-treated rats. These data demonstrate extinction impairment in the MAM model that is analogous to that observed in schizophrenia patients, that was probably due to disruption in the regulation of mPFC activity by glutamatergic neurotransmission in the BLA.
\end{abstract}

Neuropsychopharmacology (2018) 43:2459-2467; https://doi.org/10.1038/s41386-018-0128-3

\section{INTRODUCTION}

In addition to prominent positive symptoms and cognitive deficits, schizophrenia patients also exhibit alterations in emotional functions related to negative symptoms [1, 2]. The ability of schizophrenia patients to process emotional information can affect the symptom severity and contribute to psychosis [1, 3, 4]. Impairment in aversive associative learning is observed in schizophrenia [5-7], such as disruption of extinction memory [8]. Extinction involves formation of a new emotional memory, as shown by studies in rodents and humans $[9,10]$. Pavlovian fear conditioning is extensively used to understand this process; this paradigm is based on pairing a neutral stimulus as context and a tone (the conditioned stimulus-CS) with an aversive stimulus (the unconditioned stimulus-US), with the aversive stimulus being footshock in rodents [11]. After pairing, the CS can predict the US event and elicit a conditioned response, such as freezing behavior in rats [12]. The extinction of fear memory involves a decrease in the emotional response produced in response to previous aversive learning by repeated presentation of CS without the aversive stimulus, and depends on formation of new learning [10] .

Evidence in schizophrenia patients, using fear conditioning, demonstrated extinction recall impairment [8] associated with decreased ventromedial prefrontal cortex (vmPFC) function during the task [13]. vmPFC is functionally analogous to the medial prefrontal cortex (mPFC) in rodents, especially the infralimbic portion $[14,15]$. Both in humans and rodents this area is essential for extinction learning. Therefore, disruption of the mPFC directly affects the consolidation of extinction [14, 22]. The amygdala is another brain area involved in fear conditioning that has reciprocal connectivity with the $\operatorname{mPFC}[16,17]$. The balance between amygdala and MPFC is crucial in fear and extinction learning. During extinction learning the MPFC has an inhibitory role over amygdala activity and conditioned responses [17]. Recent evidence also demonstrated that the amygdala projections to the MPFC are essential for extinction learning and recall [18]. There is a limited amount of studies investigating the extinction process in animal models of schizophrenia and the neural basis of this process, especially in a developmental disruption animal model of schizophrenia.

The developmental disruption animal model of schizophrenia employed here consists of administering methylazoxymethanol acetate (MAM), a DNA alkylating agent, during gestational day 17 , which leads to a prominent increase in dopaminergic pathway of ventral tegmental area (VTA) to associative striatum in the adult. This model demonstrates substantial neuroanatomic, behavior, and electrophysiology alterations that parallel those observed in schizophrenia patients [19]. Several brain structures that are related to modulation of extinction have been shown to undergo substantial changes in this model, including the basolateral amygdala (BLA) and $\operatorname{mPFC}[20,20,51]$. The mPFC in particular

\footnotetext{
${ }^{1}$ Department of Pharmacology, Medical School of Ribeirão Preto, University of São Paulo, Ribeirão Preto/SP 14049-900, Brazil and ${ }^{2}$ Departments of Neuroscience, Psychiatry and Psychology, University of Pittsburgh, A210 Langley Hall, Pittsburgh, PA 15260, USA

Correspondence: D. L. Uliana (danielalescano@usp.br)
}

Received: 8 March 2018 Revised: 1 June 2018 Accepted: 17 June 2018

Published online: 23 June 2018 
presents significant alterations in activity $[20,51]$ that is believed to contribute to BLA hyperactivity [21] and deficits in extinction $[22,23]$. Considering that MAM-treated animals have altered mPFC and BLA activity and that these brain areas are essential in extinction memory, we examined whether MAM-treated rats also exhibit deficits in extinction memory that correspond to changes in $\mathrm{MPFC}-\mathrm{BLA}$ interactions.

\section{MATERIALS AND METHODS}

\section{Animals and methylazoxymethanol treatment}

Pregnant female Sprague-Dawley rats (Envigo, Indianapolis, USA) were acquired on GD 14 and each dam was housed individually in ventilated plastic breeding tubs. The dams were administered saline (1 mg/kg) or MAM (20 mg/kg, i.p.) on GD 17 [24]. Male offspring were weaned on postnatal day (PND) 22 and were housed in groups of two or three with littermates until approximately 3-4 months of age. Rats were then used for behavioral and electrophysiological experiments after PND 65, as young adults [25]. The rats were housed in a temperaturecontrolled room $\left(22 \pm 1{ }^{\circ} \mathrm{C}\right)$ under standard housing conditions with free access to food and water and a $12 \mathrm{~h}$ light/ dark cycle (lights on at 7 a.m.). Seven days before PDN65, the animals were transferred to a reverse light cycle room (lights on 7 p.m.). The rats were tested during the lights-off cycle in behavior experiments.

All the experiments were performed in accordance with the $\mathrm{NIH}$ Guide for the Care and Use of Laboratory Animals and approved by the Institutional Animal Care and Use Committee at the University of Pittsburgh.

\section{Contextual fear conditioning}

The conditioning chamber used was a grid floor with stainless steel rods plugged into a shock generator (MedAssociates). The animals were placed into the footshock chamber and, after 2 min habituation, four footshocks $(0.75 \mathrm{~mA}$ for $1 \mathrm{~s}$ at $20-60 \mathrm{~s}$ random intervals) were delivered. Twenty-four hours later the animals were re-exposed to the experimental chamber for a 30-min-long session ( 6 blocks of $5 \mathrm{~min}$ ) without shock delivery (extinction session). On the next day, the test (extinction recall) was recorded during $10 \mathrm{~min}$ of novel exposure. Freezing behavior was recorded during all sessions of conditioning, extinction, and test. Freezing behavior was manually recorded during sessions by an experimenter, and was defined as the complete absence of movement other than respiration while the animal assumed a characteristic splayed posture [12]. Four litters were used: Saline (4 and 3 offspring of two litters) and MAM (5 and 3 offspring of two litters) groups. Previous studies showed that litter or rearing by the injected dam did not impact the results [24].

\section{Tone fear conditioning}

The tone fear conditioning protocol was based on Brugos-Robles et al. (2007). The same chamber used in contextual fear conditioning was used in the tone protocol. In the acquisition phase, the animals were placed into the footshock chamber and after 2 min received five habituation tones $(30 \mathrm{~s} ; 65 \mathrm{~dB} ; 30-60 \mathrm{~s}$ random interval), followed by seven tones that co-terminated with footshocks $(0.5 \mathrm{~s} ; 0.6 \mathrm{~mA} ; 30-60$ random interval). Twenty-four hours later the animals were re-exposed to the chamber with 2 min baseline and 20 tone presentations (extinction session). The following day the rats were tested with $2 \mathrm{~min}$ baseline and eight tone presentations. The same chamber was used in all the sessions. Seven litters were used: Saline $(4,3,3$, and 4 offspring of four litters) and MAM (2, 2 and 3 offspring of three litters) groups.

\section{Electrophysiological recordings}

The electrophysiological experiments were performed the day after the tone fear conditioning protocol, as described previously [20]. The rats were injected with chloral hydrate $(400 \mathrm{mg} / \mathrm{kg})$ and fixed in a stereotaxic frame (Kopf). Body temperature was held constant at $37^{\circ} \mathrm{C}$ using a thermostatically-controlled heating pad (Fintronics). Anesthesia was maintained by i.p. injection of chloral hydrate as needed to maintain suppression of the hindlimb compression reflex. In vivo extracellular recordings were performed using microelectrodes pulled from Omegadot $2.0 \mathrm{~mm}$ glass tubing on a vertical electrode puller (Narishige P-5, Japan) and the tip broken back under microscopic control to an impedance of $12-16 \mathrm{M} \Omega$ and filled with $2 \mathrm{M} \mathrm{NaCl}$ containing $2 \%$ Sky Blue dye. Microelectrodes were lowered through the MPFC (A/ $\mathrm{P}+3.2-2.4 \mathrm{~mm}$ from bregma; $\mathrm{M} / \mathrm{L}+0.4-0.5 \mathrm{~mm}$ from midline; $\mathrm{V}$ -2.5 to $4.5 \mathrm{~mm}$ from brain surface). Electrophysiological signals were amplified $(\times 1000)$ and filtered with $10-50 \mathrm{~Hz}$ high pass and $16 \mathrm{kHz}$ low pass using an amplifier (model WDR-420). An oscilloscope (B\&K precision) was used to display the electrophysiological signal and the data were transferred via a Powerlab interface ( $A D$ instruments) to a computer with LabChart v. 8 software. A signal-to-noise ratio $>3$ was the cutoff used for data analyses.

Concentric bipolar stimulation electrodes (NEX-100X; Rhodes Medical Instruments) positioned in the BLA (A/P $-2.6 \mathrm{~mm}$ from bregma; $\mathrm{M} / \mathrm{L}-4.6$ to $4.8 \mathrm{~mm}$ from midline; $\mathrm{V}-7.8$ to $8 \mathrm{~mm}$ from skull) were used to apply the single-pulse and high frequency stimulation (HFS; $20 \mathrm{~Hz} ; 10 \mathrm{~s}$ at suprathreshold). Single-pulse stimulation in the BLA was applied using a dual-output stimulator (S8800; Grass Technologies) with $1 \mathrm{~mA}$ intensity/0.5 Hz frequency/ $0.25 \mathrm{~ms}$ pulse duration to search for a responsive neuron in the mPFC. When a responsive short latency monosynaptically activated neuron was found the current administered to the BLA was adjusted to evoke an action potential $50 \%$ of the time. A response was considered to be monosynaptic when the latency to the evoked action potential was approximately $22 \mathrm{~ms}$ and when increasing stimulus intensity resulted in little shift in latency [26]. Spike probability was measured $10 \mathrm{~min}$ before HFS (baseline) and 30 min after HFS, dividing the number of spikes by the total number of single-pulse stimuli. Only 1 neuron was recorded per animal. The electrophysiological recordings in conditioned rats were performed in the same offspring and litters as described in tone fear conditioning. The experiment with $\mathrm{PL}$ and $\mathrm{IL}$ region comparison in Saline groups used four litters (PL: 3, 2, 1, and 2 offspring for each litter; IL: 1, 1, 2, and 2 offspring for each litter). The experiments with naive saline and MAM-treated rats were derived from four litters (Saline, 5 and 3 offspring of two litters; MAM, 4 and 2 offspring of two litters).

\section{BLA cannula implantation and drug administration}

For experiment with intra-BLA drug administration, two litters that were treated with saline on GD17 were used (Saline group, three offspring of each litter; Drug administration group, four and three offspring of litters). For implantation of cannulae into the BLA, rats were anesthetized with isoflura6ne and fixed in the stereotaxic frame. After incision, stainless steel guide cannulae were implanted bilaterally targeting the BLA (A/P $-2.8 \mathrm{~mm}$ from bregma; $M / L+5.0 \mathrm{~mm}$ from midline; $V-7.8 \mathrm{~mm}$ from skull) according to Paxinos and Watson (1997). Acrylic cement and one metal screw were used to fix it to the skull. After surgery, a subcutaneous injection of a nonsteroidal anti-inflammatory (Carprofen; Rimadyl, Zoetis Inc) was administered for postoperative analgesia, and a medicated food (MediGel ${ }^{\circledR} \mathrm{CPF}$ ) was provided in the rat homecage. To survey recovery the rats were maintained in an isolated environment for 2 days, after which the rats were housed in groups of two with a littermate in a clean cage. The rats were maintained in the animal care unit for 7 days to complete recovery before initiating behavior experiments.

All rats received similar exposure to the tone conditioning session. One day after, the rats were randomly assigned to saline or treatment groups. Ten minutes before the extinction session saline $0.9 \%(0.5 \mu \mathrm{l})$ or NMDA antagonist (DL-2-amino-5- 
phosphonopentanoic acid, AP5 $5.07 \mathrm{mM} / \mathrm{L}$ or $2.5 \mathrm{nmol} / 0.5 \mu \mathrm{l}$ per injection; Sigma) [27] was administered into the BLA using a $2 \mu \mathrm{l}$ Hamilton syringe (Hamilton Co., USA). After injection, the needles remained in place for an additional $30 \mathrm{~s}$ to prevent reflux. The animals were then tested for extinction using tone fear conditioning. Twenty-four hours later the groups of animals were submitted to test of extinction recall.

The dose of $2.5 \mathrm{nmol}$ per site of NMDA antagonist was based on a dose that should be adequate to impair extinction using tone fear conditioning [27].

\section{Histology}

At the end of electrophysiological recordings, the electrode location (recording site) was marked with electrophoretic ejection of Chicago Sky Blue dye $(-20 \mu \mathrm{A}$ constant negative current, 20 min). The location of the stimulation electrode was marked by administering $10 \mathrm{~s}$ cathodal current at $200 \mu \mathrm{A}$. A lethal dose of chloral hydrate (additional $400 \mathrm{mg} / \mathrm{kg}$ ) was administered for euthanasia. In rats that received the BLA implantation, after testing the animals were killed using a closed chamber and $\mathrm{CO}_{2}$.

The rats were decapitated and the brains removed before fixing the tissue in $8 \%$ paraformaldehyde for approximately $48 \mathrm{~h}$, following by transferring to $25 \%$ sucrose solution for cryoprotection. The brains were frozen and sliced coronally $(60 \mu \mathrm{m})$, after saturation, using a cryostat (Leica Frigocut 2800). The slices were mounted on gelatin-chormalum-coated slides and stained with a combination of neutral red and cresyl violet.

\section{Statistical analyses}

Freezing behavior in conditioning/extinction sessions and electrophysiological recording data were analyzed using a two-way ANOVA (Time-repeated factor; condition-independent variable and freezing behavior/\%change in spike probability-dependent variable). The extinction retention test and spike probability were analyzed using the Student's $t$-test (Condition-independent variable and freezing behavior/spike probability-dependent variable). All data were represented as the mean \pm SEM. Statistical tests with $p<0.05$ were considered significant.

\section{RESULTS}

Contextual fear conditioning

In the acquisition session of contextual fear conditioning, saline ( $n$ $=7)$ and MAM rats $(n=8)$ acquired the contextual fear memory, and no difference was observed between the groups (Fig. 1a; twoway ANOVA; time $F_{4.52}=176.2, p<0.05$; condition $p>0.05$; interaction $p>0.05)$. On day two, saline $(n=7)$ and MAM groups $(n=8)$ were similar in contextual fear expression (first point of extinction, $p>0.05, t$-test). In the extinction session, no difference was found between both groups (saline $n=7$ and MAM $n=8$; two-way ANOVA; time $F_{5.65}=25, p<0.05$; condition $p>0.05$; interaction $p>0.05$ ). No difference was observed between saline $(n=7)$ and MAM groups $(n=8)$ in extinction recall $t(p>0.05, t$ test).

Tone fear conditioning

Saline $(n=14)$ and MAM $(n=7)$ groups acquired the tone fear memory conditioning at the same rate (Fig. $1 \mathrm{~b}$; time, $F_{5.95}=700.5$; condition, $p>0.05$; interaction, $p>0.05$; two-way ANOVA). During the extinction session, baseline and fear expression (first point of training) were similar between saline $(n=14)$ and MAM groups ( $n$ $=7)$ ( $p>0.05, t$-test). Two-way ANOVA showed a significant effect of time $\left(F_{9.17}=18.47, p<0.05\right)$, condition $\left(F_{1.19}=5.52, p<0.05\right)$, but there was no interaction $(p>0.05)$ in the extinction session. In extinction recall, saline $(n=14)$ and MAM-treated animals $(n=7)$ were significantly different at baseline $(t=2.24, p<0.05)$ and during the extinction recall $(t=2.96, p<0.05)$.
BLA-mPFC plasticity

In conditioned saline and MAM groups, single-pulse stimulation of the BLA was use to evoke spike discharge in MPFC neurons (Fig. $2 a-c)$. The number of neurons recorded was 14 in salinetreated and seven in MAM-treated groups. The mean baseline spike probability was not different between groups $(p>0.05, t$ test, Fig. 2d). The current used to obtain at least $50 \%$ spike probability baseline for BLA-evoked response was not significantly different between saline $(n=14)$ and MAM-treated $(n=7)$ animals (Saline $1.12 \mathrm{~mA} \pm 0.031$ and MAM $1.09 \mathrm{~mA} \pm 0.035 ; p>0.05, t$-test;). At baseline, there was no difference in the number of neurons that demonstrate burst activity evoked by single-pulse stimulation of the BLA (saline, $n=14,42.9 \%$ and MAM, $n=7,37.5 \%$ of total neurons recorded). The latency to onset of the evoked action potential was not significantly different between the groups (saline, $n=14,19.65 \mathrm{~ms} \pm 1.132$ and MAM, $n=7,17.60 \mathrm{~ms} \pm 1.885$; $p>0.05$, t-test). BLA HFS in saline-treated animals $(n=14)$ decreased probability of evoking spikes in MPFC neurons, which was not observed in MAM-treated animals $(n=7)$ (2-way ANOVA significant effect of time $\left(F_{7.13}=2.76, p<0.05\right)$, condition $\left(F_{1.19}=\right.$ $10.01, p<0.05)$ and interaction $\left(F_{7.13}=2.34, p<0.05\right.$; Fig. $\left.2 \mathrm{e}\right)$. The mean percent change in BLA-evoked spike probability following HFS was decreased in saline rats $(n=14)$, but not in the MAM group $(n=7)(t=2.99, p<0.05, t$-test, Fig. $2 f)$.

In saline animals, BLA stimulation produced similar spike probabilities with $\mathrm{PL}(n=8)$ and IL $(n=6)$ stimulation $(p>0.05$, $t$-test, Fig. 3a-c; significant effect of time, $F_{7.84}=7.52, p<0.05$, but not mPFC subregion, $p>0.05$, Fig. $3 \mathrm{~d}$ ). The mean \%Change in BLAevoked spike probability was not significantly different comparing $\mathrm{PL}(n=8)$ and IL $(n=6)$ portions $(p>0.05$; Fig. $3 \mathrm{e})$.

In order to examine whether conditioning impacted spike probability, the impact of single-pulse and HFS of BLA in naive animals on the mPFC was evaluated. The number of neurons recorded was eight in saline-treated and six in MAM-treated groups. With single-pulse stimulation at baseline, saline and MAMtreated animals did not exhibit differences in evoked spike probability ( $p>0.05, t$-test, Fig. $4 c)$ and intensity of current required to obtain at least $50 \%$ spike probability $(p>0.05, t$-test; $n=8,1.27 \pm 0.044 ;$ MAM, $n=6,1.28 \pm 0.036$ ). The latency to onset of the evoked action potential was not significantly different between the groups (saline, $n=8,22.34 \mathrm{~ms} \pm 1.577$ and MAM, $n$ $=6,18.25 \mathrm{~ms} \pm 1.639 ; p>0.05, t$-test). BLA HFS in saline rats $(n=8)$ also decreased the probability of evoking spikes in MPFC neurons, which was not observed in the MAM rats $(n=6)$ (2-way ANOVA significant effect of time $\left(F_{7.84}=4.63, p<0.05\right)$, condition $\left(F_{1.12}=\right.$ $5.71, p<0.05)$ and interaction $\left(F_{7.84}=3.46, p<0.05\right.$; Fig. $\left.4 d\right)$. The mean percent change in BLA-evoked spike probability was significantly decreased in the saline group $(n=8)$ compared to MAM-treated rats $(n=6)(t=2.39, p<0.05, t$-test, Fig. $4 \mathrm{e})$.

NMDA antagonist injection in BLA

To investigate whether the BLA itself was involved in the differential impact of BLA stimulation on mPFC plasticity, an NMDA antagonist (AP5; $2.5 \mathrm{nmol} / 0.5 \mu \mathrm{l}$ ) was administered into the BLA before extinction (Fig. 5). Both groups of rats acquired fear conditioning (saline $n=6$ and MAM $n=7$; time effect, $F_{5.55}=$ 255.2, $p<0.05$; group effect $p>0.05$; interaction, $p>0.05$; two-way ANOVA). In the extinction session, no difference was observed at baseline ( $p>0.05$; $t$-test). However, the rats that received NMDA antagonist $(n=7)$ did not extinguish the fear response $(p>0.05$, ANOVA), as was observed in the saline group $\left(n=7 ; F_{9.50}=2.60, p\right.$ $<0.05$, repeated measures ANOVA effect of time $\left(F_{9.99}=7.08, p<\right.$ $0.05)$, treatment $\left(F_{1.11}=8.05, p<0.05\right)$ and no interaction $(p<$ $0.05)$. During the test session, the rats that received the NMDA antagonist $(n=7)$ exhibited a higher degree of freezing at baseline $(t=2.86, p<0.05$; $t$-test) and at testing $(t=3.31, p<$ 0.05 ; $t$-test) compared to saline group $(n=6)$. 


\section{A Contextual fear conditionig}

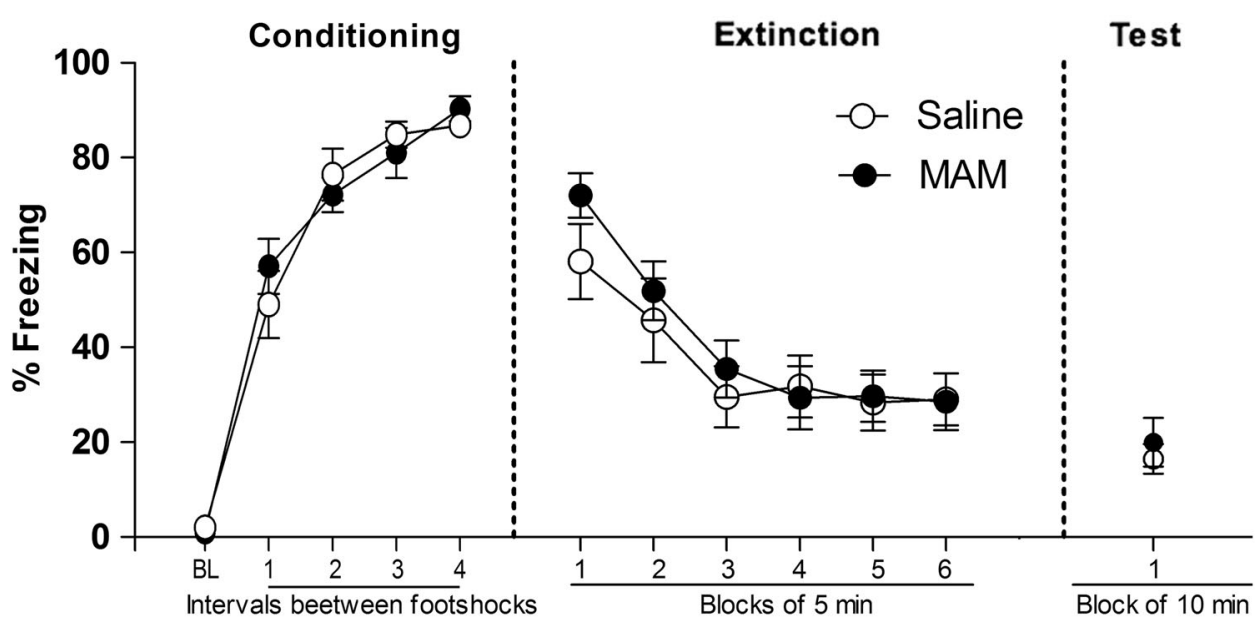

B Tone fear conditioning

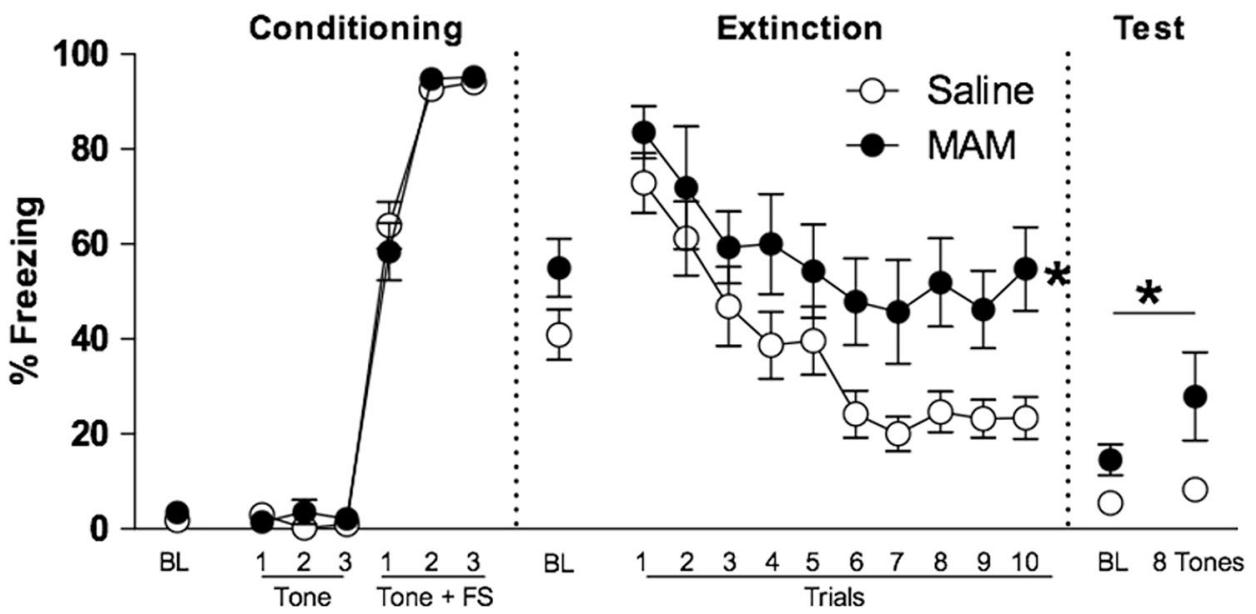

Fig. 1 MAM rats do not show differences in contextual fear conditioning, but demonstrate deficits in extinction learning and recall in tone fear conditioning. a Contextual fear conditioning in saline-treated ( $n=7$; white circles) and MAM-treated ( $n=8$; black circles) rats. Conditioning session (4 FS of $0.75 \mathrm{~mA} / 1 \mathrm{~s}$ ) in saline-treated and MAM-treated rats represents acquisition of fear memory. Extinction (6 blocks of $5 \mathrm{~min} ; 30 \mathrm{~min}$ total) and test $(10 \mathrm{~min})$ sessions in both group represent extinction learning and recall. No statistic difference was observed between MAM and saline rats in any session. b Impairment of extinction learning and recall in MAM-treated rats. Tone fear conditioning (7Tone + FS $0.6 \mathrm{~mA} / 0.5 \mathrm{~s}$ ) in saline-treated ( $n=14$; white circles) and MAM-treated ( $n=7$; black circles) rats. In the conditioning session, no difference was observed between saline-treated and MAM-treated rats. In the extinction session (10 trials; 20 total tones), no difference was observed at baseline, but an impairment of extinction learning was observed ( ${ }^{*} p<0,05$ to saline group). In the test session ( 8 tones) there was a difference at baseline and with extinction recall between saline-treated and MAM-treated rats $\left({ }^{*} p<0.05\right)$

\section{DISCUSSION}

Prenatal MAM treatment, as described previously, produces a set of alterations that is analogous to those observed in schizophrenia patients [19], such as deficits in social interaction and spatial memory $[24,28]$. In humans, schizophrenia patients present impairment of extinction recall memory in fear conditioning [8], suggesting that aversive learning is abnormal. Using tone fear conditioning we first described an alteration in aversive learning in MAM rats. Thus, MAM-treated rats exhibit a deficit in tone fear extinction learning and recall, demonstrating an abnormal processing of extinction learning that partially mimics what happens in schizophrenia patients. Moreover, we demonstrated that MAM-treated rats have no alteration in contextual fear conditioning. A recent study from our group suggests that MAM-treated animals have a deficiency in context discrimination to predict a future aversive situation [29]. In the contextual fear conditioning experiment, we observed a similar expression of conditioned response between saline and MAMtreated animals. This difference is probably due to the different protocol used, where in the present study we applied a higher number of footshocks (4) and higher intensity $(0.75 \mathrm{~mA})$ as contrasted with one footshock $(0.5 \mathrm{~mA})$ in the previous study that probably produced a strong association with context. In both protocols, the MAM-treated animals did not demonstrate impairment in acquisition. Schizophrenia patients are reported to exhibit a deficit in acquisition of emotional memories using a different task [30]. The fear conditioning task used here likely represents a simplified task, especially with respect to the contextual protocol, and was not capable of revealing the disruption in acquisition of conditioned memory. However, the present results demonstrated a significant impairment in extinction using tone fear conditioning that reflects the robust 
A

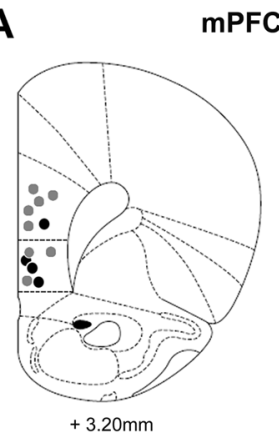

MPFC

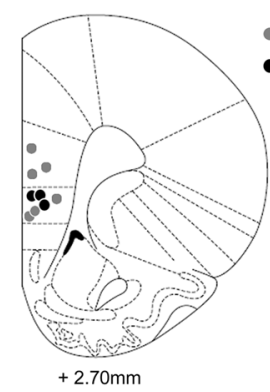

C

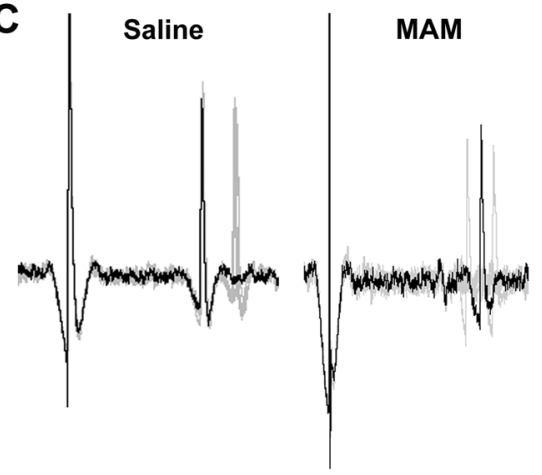

$\mathbf{E}$

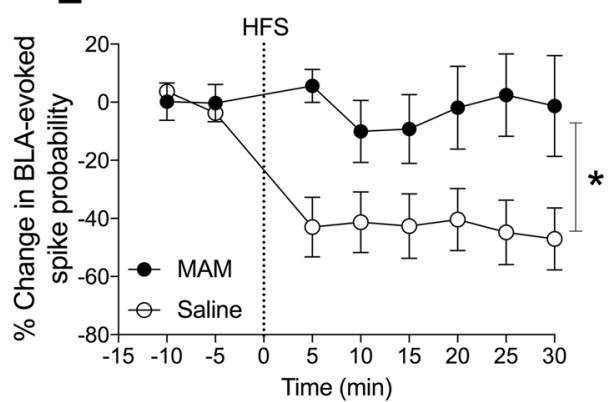

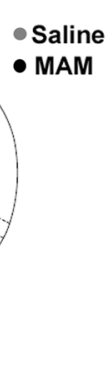

B

\section{B}

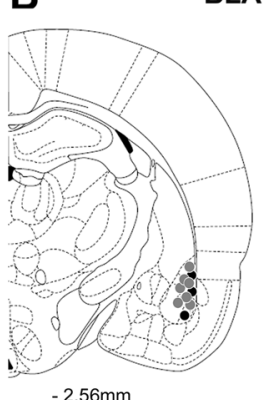

$-2.56 \mathrm{~mm}$
BLA
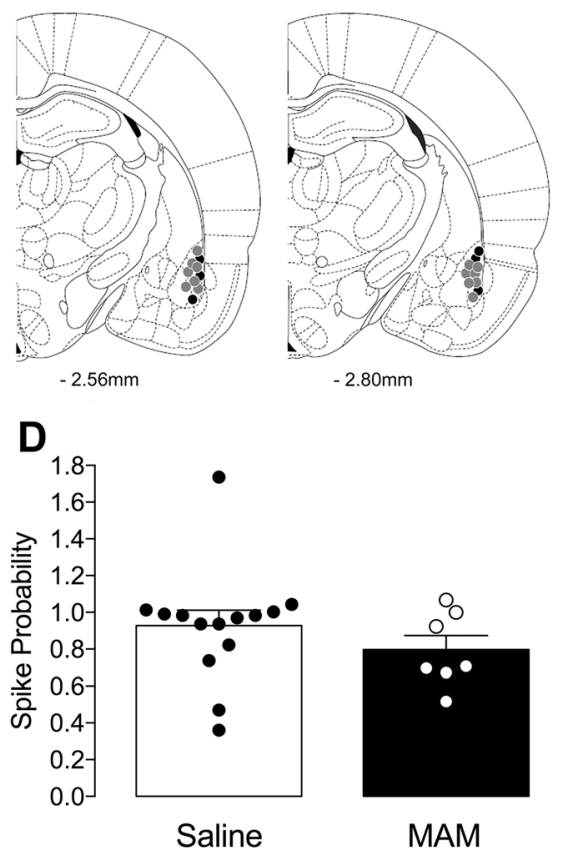

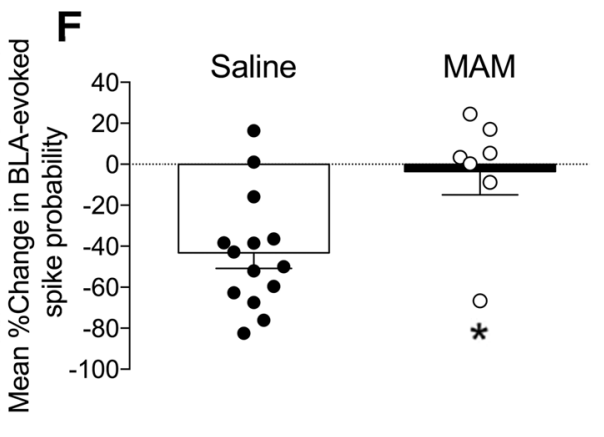

Fig. 2 HFS of the BLA induces abnormal plasticity in the MPFC in MAM-treated rats. a Schematic illustration of recording electrode placement in $\mathrm{MPFC}$ and $\mathbf{b}$ stimulation electrodes in BLA for saline-treated (gray circles; $n=14$ ) and MAM-treated (black circles; $n=7$ ) rats, as shown in coronal sections of rat brain taken from Paxinos and Watson [48]. c Representative example of extracellular mPFC recording showing a neuron responsive to single BLA stimulation $(1 \mathrm{~mA})$ in saline-treated and MAM-treated animals. d Spike probability over 10 min in saline-treated and MAM-treated rats evoked by $1 \mathrm{~mA}$ stimulation. e MAM-treated rats (black circles) showed significantly less change in BLA-evoked spike probability following HFS of the BLA compared to saline-treated (white circles) rats. $\left({ }^{*} p<0.05\right)$. f MAM-treated rats showed significantly less change in BLA-evoked spike probability following HFS compared to saline-treated rats $\left({ }^{*} p<0.05\right)$

disruption of this process in MAM rats that is analogous to that observed in schizophrenia patients [8].

The fear extinction impairment demonstrated in the tone conditioning protocol was not observed in contextual conditioning. This difference could be related to different parameters used in the protocols. The tone protocol required a higher number of events (7Tone + Footshocks) to elicit a substantial fear expression in contrast to the contextual protocol (4 Footshocks). However, it's important to note that contextual relevance in the tone protocol, represented as baseline freezing before extinction, did not differ between saline and MAM-treated groups. This evidence supports the idea that fear extinction disruption in tone conditioning probably is not related to differences in the protocol. Moreover, the results with contextual and tone fear conditioning also possibly relate to different neural pathways that control these responses [17]. Evidence suggests that in fear conditioning, contextual information is preferentially modulated by the hippocampus and tone information is controlled by the amygdala [17]. In the MAM model, the alteration in amygdala activity likely has an important role in fear conditioning. MAM rats exhibit hyperactivity in the BLA [21], which could contribute to the abnormalities in extinction memory observed here. Although hyperactivity in the ventral hippocampus (vHipp) has been characterized in MAM rats [31], the vHipp in this preparation did not impact the BLA-mPFC pathway [32]. This suggests that the contextual valence in extinction that is dependent on the vHipp is not essential for learning of extinction under the conditions tested. Evidence demonstrated that BLA projections to MPFC are important in modulating the MPFC excitability and also the extinction process $[18,33,49,50]$. Optogenetic HFS of the BLAmPFC projection before extinction training has been shown to improve extinction training and recall [18]. Considering the presence of BLA hyperactivity in MAM-treated rats and the role of BLA-mPFC pathway in aversive memory, we examined whether this pathway played a role in extinction investigated here. HFS of the BLA was found to decrease the spike probability in the BLAMPFC pathway of saline-treated rats, but not in MAM rats, reflecting a deficient modulation of MPFC activity by the BLA. 
A

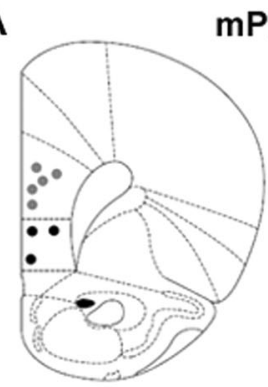

$+3.20 \mathrm{~mm}$
mPFC

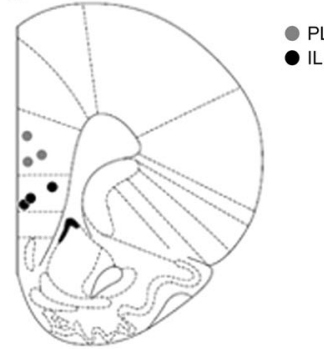

$+2.70 \mathrm{~mm}$

C $\quad 2.0-$

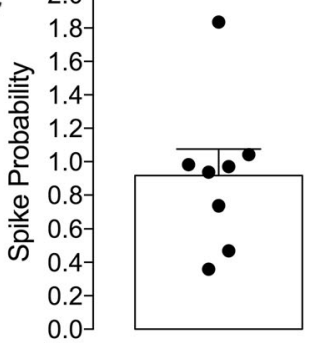

PL
B

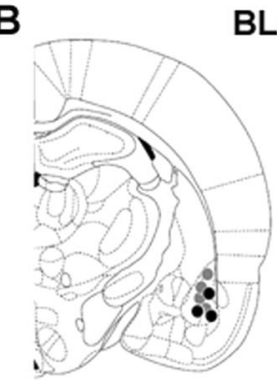

$-2.56 \mathrm{~mm}$
BLA

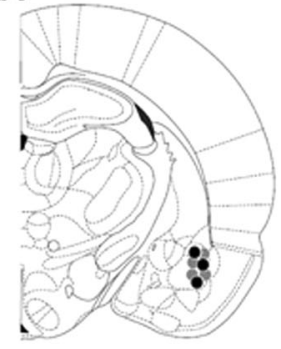

$-2.80 \mathrm{~mm}$

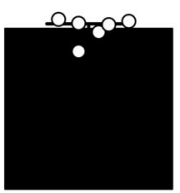

IL

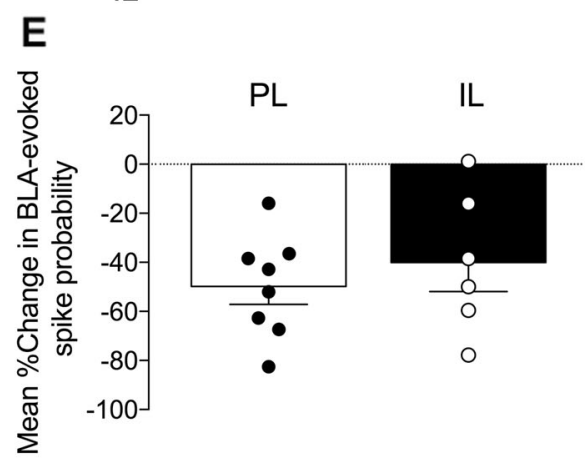

Fig. 3 HFS of BLA does not elicit significant differences in plasticity in the PL $(n=8)$ and IL $(n=6)$ subdivisions of the mPFC of saline-treated animals. a Schematic illustration of recording electrode placements in PL or IL mPFC and $\mathbf{b}$ stimulation electrodes in BLA for PL (gray circles) and IL (black circles) subdivisions illustrated in coronal sections of rat brain taken from Paxinos and Watson [48]. c Spike probability over 10 $\mathrm{min}$ in PL and IL subdivisions in saline-treated rats evoked by $1 \mathrm{~mA}$ stimulation. d Mean percent change ( \pm SEM) in BLA-evoked spike probability over the time, normalized to baseline, following HFS of the BLA and recorded in PL (white circle) and IL (black circles) of salinetreated rats. e Mean percent change in BLA-evoked spike probability following HFS in PL and IL of saline-treated

Moreover, this same response was observed in naive MAM rats, suggesting that impairment in BLA-mPFC plasticity is a condition specific to MAM-treated rats. Neuronal excitability decreases in mPFC after HFS of BLA was described recently in an in vitro study using optogenetic methodology [18], which is consistent with the current in vivo data.

Data from the electrophysiological recordings revealed a reduction in BLA-evoked spike probability in $\mathrm{MPFC}$ in normal conditions. We propose that activation of the BLA projection induces disinhibition of mPFC function by inhibiting mPFC interneurons. Thus, in the normal case, BLA activation during extinction learning could induce a gradual reduction in plasticity that would be expected to disinhibit the mPFC during the task, resulting in an efficient extinction learning and recall, considering that normal mPFC activity is essential for this type of learning [22]. This idea is consistent with results demonstrating that PVimmunoreactive GABAergic interneurons in $\mathrm{mPFC}$ receive glutamatergic projections from the BLA [34-36], supporting the proposed $\mathrm{mPFC}$ interneuron control by the BLA projections in our study. In MAM-treated animals, however, BLA activation during this task is impaired, likely due to PV interneuron dysfunction [37, 52], which would produce an opposite action of the BLA on MPFC plasticity and disruption of extinction learning and recall. These results are in accordance with a previous study in MAM-treated animals demonstrating that activity of the mPFC during a latent inhibition paradigm is deficient, suggesting a disruption of GABAergic signaling during the task [37]. Moreover, MAM-treated animals show decreased expression of PV-positive interneurons in MPFC [37] that reflect a GABAergic dysfunction in the mPFC of MAM rats. Thus, the impairment in MPFC interneuron function would interfere with the BLA-mPFC pathway and impaired extinction learning. Additional evidence also demonstrated that MPFC interneurons act to direct CS attention [38] and if this interneuron activity is disrupted, as observed in mPFC of MAM-treated rats, it would likely impact both the conditioned response and extinction learning. It has been demonstrated that schizophrenia patients exhibit deficiency in activation of mPFC under a cognitive task $[39,40]$, which is consistent with the cortical inability to control cognitive functions as observed in animal models.

Evidence for an opposite role of the $\mathrm{PL}$ and $\mathrm{IL}$ portion of the mPFC in aversive response has been described [41-43]. In fear conditioning, PL activity is related to expression of fear conditioning response and IL activity is essential for extinction 
A

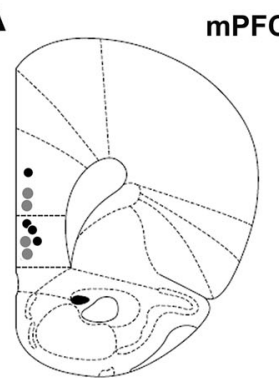

$+3.20 \mathrm{~mm}$

mPFC

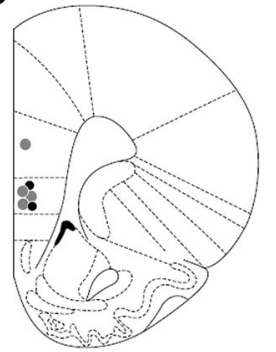

$+2.70 \mathrm{~mm}$
B

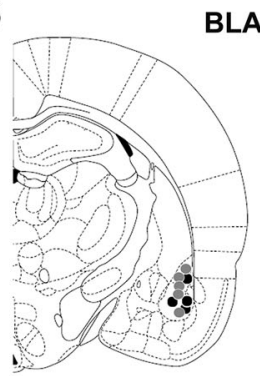

$-2.56 \mathrm{~mm}$

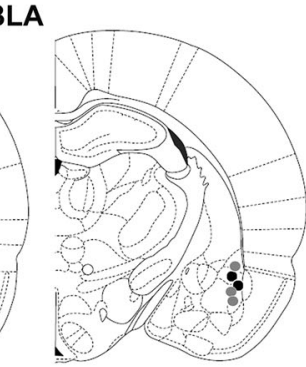

$-2.80 \mathrm{~mm}$

C

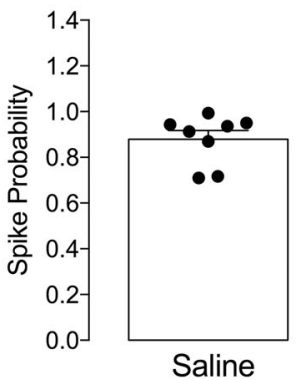

D

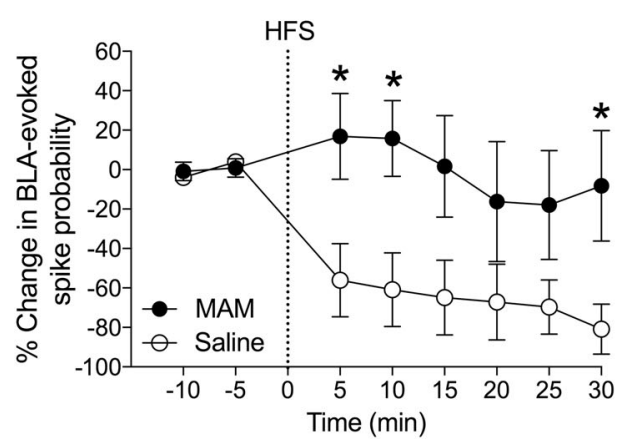

$\mathbf{E}$

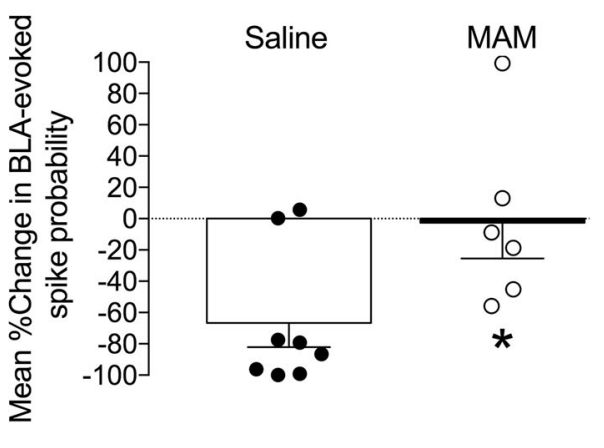

Fig. 4 HFS of the BLA induces abnormal plasticity in the MPFC in naive MAM-treated rats. a Schematic illustration of recording electrode placement in $\mathrm{mPFC}$ and $\mathbf{b}$ stimulation electrodes in BLA for saline-treated (gray circles; $n=8$ ) and MAM-treated (black circles; $n=6)$ rats, as shown in coronal sections of rat brain taken from Paxinos and Watson [48]. c Spike probability over 10 min in naive saline-treated and MAMtreated rats evoked by $1 \mathrm{~mA}$ stimulation. d Naive MAM-treated rats (black circles) showed significantly less change in BLA-evoked spike probability following HFS of the BLA compared to naive saline-treated (white circles) rats. ( $\left.{ }^{*} p<0.05\right)$. e Naive MAM-treated rats showed significantly less change in BLA-evoked spike probability following HFS compared to naive saline-treated rats $\left({ }^{*} p<0.05\right)$

learning [22]. In our study, PL and IL neurons presented a similar response; i.e., a global decrease of spike probability after BLA HFS. However, this result does not exclude that PL and IL subdivisions present opposite responses in neuronal activity during fear extinction training in saline-treated rats. Differential roles of $\mathrm{PL}$ and IL have also been suggested in response to stressful events [41, 42]. Thus, the PLPFC has an inhibitory effect on the response to stress $[44,45]$ whereas the IL facilitates this effect $[42,43]$. The PL is impacted by both amygdala and hippocampus activity during stressors that trigger emotional responses $[41,46]$. It is possible that during conditioning the $\mathrm{PL}$ dysfunction in MAM-treated animals produces a strong association of fear memory, with greater activation of the amygdala. Inefficient inhibition of the BLA by IL during extinction secondary to interneuron loss may also contribute to extinction disruption. It is therefore likely that this condition facilitated the pro-aversive emotional learning.

The amygdala is a key area for the control of fear and extinction memory. Therefore, we propose that dysfunction in the BLA could be a mechanism by which MAM-treated animals exhibit extinction impairment. As described previously, the BLA sends glutamatergic projections to the mPFC that provide MPFC disinhibition [34-36].
In fear conditioning, NMDA antagonist delivered into the BLA before the extinction session was described to negatively impact extinction learning [27]. However, in that study the extinction recall was not tested. In our study, NMDA antagonist injected before the extinction session produces impairment in extinction learning and a greater response during the test session. This response demonstrated that glutamatergic transmission within the BLA is essential to extinction training and the disruption of this neurotransmission affected extinction events. The impairment in glutamate signaling could be related to events observed in animal models of psychiatric disorders, as characterized in the MAM model. Glutamatergic transmission in the BLA probably is controlled by local projections within amygdala subnuclei (i.e., central and lateral nuclei) [47] that exhibit reciprocal connections with the MPFC [16]. The glutamatergic transmission that is altered by NMDA antagonist infusion into the BLA demonstrates an important bidirectional regulation of the amygdala in the modulation of extinction responses. Moreover, these data are similar to that observed in MAM-treated animals in fear conditioning. This similar response between MAM-treated animals and the group of animals treated with NMDA antagonist into the BLA before extinction suggests that glutamatergic neurotransmission 

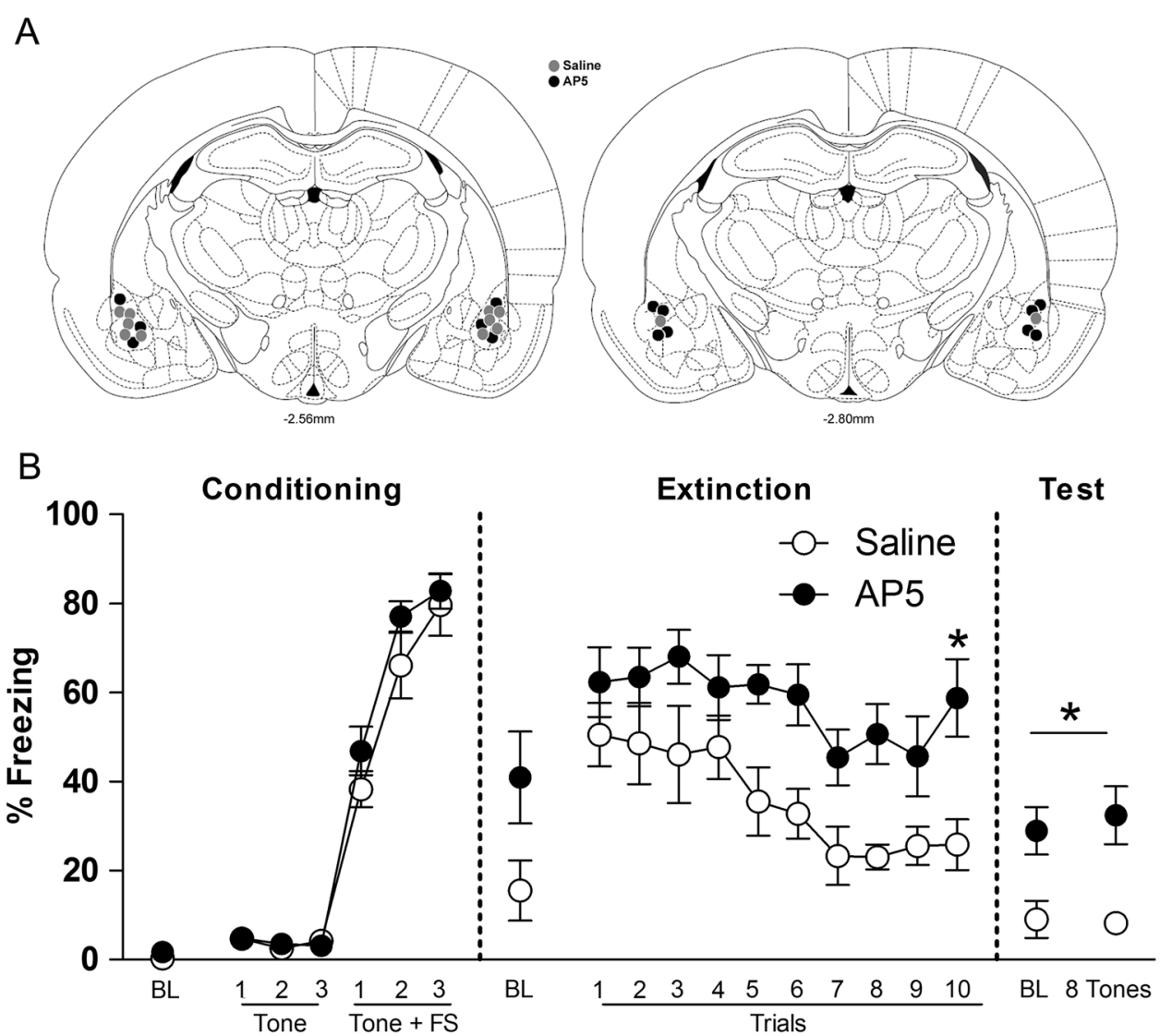

Fig. 5 Extinction impairment following NMDA antagonist (AP5; $2.5 \mathrm{nmol} / 0.5 \mu \mathrm{l}$ ) injection into the BLA before the extinction session in Salinetreated rats. a Schematic illustration of injection sites in BLA for saline (gray circles) and NMDA antagonist (black circles) groups in coronal sections of rat brain taken from Paxinos and Watson [48]. b Tone fear conditioning (7Tone + FS $0.6 \mathrm{~mA} / 0.5 \mathrm{~s}$ ) following saline ( $n=6$; white circles) and NMDA antagonist ( $n=7$; black circles) injection into the BLA before extinction in saline-treated rats [49, 50]. In the conditioning session, no difference was observed between saline and NMDA antagonist groups. During extinction (10 trials; 20 total tones), no difference in baseline was observed; however, an impairment in extinction learning was observed in the AP5 treated group ( ${ }^{*} p<0,05$ to saline group). In the test session ( 8 tones) the AP5 treated groups exhibited a significant impairment at baseline and with extinction recall $\left({ }^{*} p<0.05\right.$ to saline group)

within the BLA could be one transmission involved in extinction impairment observed in MAM-treated animals.

Collectively, our results demonstrate that MAM-treated rats have a significant disruption of extinction learning and recall using tone fear conditioning, probably due to alteration in the BLAmPFC projection. We propose that glutamatergic projections from the BLA to the MPFC did not activate efficiently the interneurons in the mPFC that in normal conditions disinhibit mPFC activity, which in turn affects extinction. Moreover, these results demonstrate the translational nature of using the MAM model of schizophrenia to examine the circuits that disrupt aversive learning in schizophrenia patients.

\section{ACKNOWLEDGEMENTS}

The authors wish to thank Niki MacMurdo and Christy Smolak for technical assistance, Kathryn Gill and Felipe Gomes for technical support. This study was supported by the Brazilian Federal Agency for Support and Evaluation of Graduate Education, PDSE/CAPES (88881.135250/2016-01) and US National Institutes of Health (MH57440 to A.A.G.).

\section{ADDITIONAL INFORMATION}

Competing interests: AAG has received funds from Lundbeck, Pfizer, Otsuka, Lilly, Roche, Asubio, Abbott, Autofony, Janssen, Alkermes, Newron, Takeda. The other authors declare no competing interests.
Publisher's note: Springer Nature remains neutral with regard to jurisdictional claims in published maps and institutional affiliations.

\section{REFERENCES}

1. Polgár $P$, Farkas $M$, Nagy $O$, Kelemen $O$, Réthelyi J, Bitter I, Myers CE, Gluck MA, Kéri S. How to find the way out from four rooms? The learning of "chaining" associations may shed light on the neuropsychology of the deficit syndrome of schizophrenia. Schizophr Res. 2008;99:200-7.

2. Waltz JA, Frank MJ, Wiecki TV, Gold JM. Altered probabilistic learning and response biases in schizophrenia: behavioral evidence and neurocomputational modeling. Neuropsychology. 2011;25:86-97.

3. Barch DM, Dowd EC. Goal representations and motivational drive in schizophrenia: the role of prefrontal-striatal interactions. Schizophr Bull. 2010;36:919-34.

4. Strauss GP, Frank MJ, Waltz JA, Kasanova Z, Herbener ES, Gold JM. Deficits in positive reinforcement learning and uncertainty-driven exploration are associated with distinct aspects of negative symptoms in schizophrenia. Biol Psychiatry. 2011;69:424-31.

5. Hofer E, Doby D, Anderer P, Dantendorfer K. Impaired conditional discrimination learning in schizophrenia. Schizophr Res. 2001;51:127-36.

6. Jensen J, Willeit M, Zipursky RB, Savina I, Smith AJ, Menon M, Crawley AP, Kapur S. The formation of abnormal associations in schizophrenia: neural and behavioral evidence. Neuropsychopharmacology. 2008;33:473-9.

7. Kosmidis $\mathrm{MH}$, Breier A, Fantie BD. Avoidance learning in schizophrenia: a dissociation between the effects of aversive and non-aversive stimuli. Schizophr Res. 1999;38:51-9. 
8. Holt DJ, Lebron-Milad K, Milad MR, Rauch SL, Pitman RK, Orr SP, Cassidy BS, Walsh JP, Goff DC. Extinction memory is impaired in schizophrenia. Biol Psychiatry. 2009;65:455-63.

9. Furini C, Myskiw J, Izquierdo I. The learning of fear extinction. Neurosci Biobehav Rev. 2014;47:670-83.

10. Milad MR, Quirk GJ. Fear extinction as a model for translational neuroscience: ten years of progress. Annu Rev Psychol. 2012;63:129-51.

11. Izquierdo I, Furini CRG, Myskiw JC. Fear memory. Physiol Rev. 2016;96:695-750.

12. Fanselow MS. Conditioned and unconditional components of post-shock freezing. Pavlov J Biol Sci. 1980;15:177-82.

13. Holt DJ, Coombs G, Zeidan MA, Goff DC, Milad MR. Failure of neural responses to safety cues in schizophrenia. Arch Gen Psychiatry. 2012;69:893-903.

14. Milad MR, Wright Cl, Orr SP, Pitman RK, Quirk GJ, Rauch SL. Recall of fear extinction in humans activates the ventromedial prefrontal cortex and hippocampus in concert. Biol Psychiatry. 2007;62:446-54.

15. Phelps EA, Delgado MR, Nearing KI, LeDoux JE. Extinction learning in humans: role of the amygdala and vmPFC. Neuron. 2004;43:897-905.

16. Marek R, Strobel C, Bredy TW, Sah P. The amygdala and medial prefrontal cortex: partners in the fear circuit. J Physiol. 2013;591:2381-91.

17. Quirk GJ, Mueller D. Neural mechanisms of extinction learning and retrieval. Neuropsychopharmacology. 2008;33:56-72.

18. Klavir O, Prigge M, Sarel A, Paz R, Yizhar O. Manipulating fear associations via optogenetic modulation of amygdala inputs to prefrontal cortex. Nat Neurosci. 2017;20:836-44.

19. Modinos G, Allen P, Grace AA, McGuire P. Translating the MAM model of psychosis to humans. Trends Neurosci. 2015;38:129-38.

20. Belujon P, Patton MH, Grace AA. Role of the prefrontal cortex in altered hippocampal-accumbens synaptic plasticity in a developmental animal model of schizophrenia. Cereb Cortex. 2014;24:968-77.

21. Du Y, Grace AA. Amygdala hyperactivity in MAM model of schizophrenia is normalized by peripubertal diazepam administration. Neuropsychopharmacology. 2016;41:2455-62.

22. Quirk GJ, Garcia R, González-Lima F. Prefrontal mechanisms in extinction of conditioned fear. Biol Psychiatry. 2006;60:337-43.

23. Burgos-Robles A, Vidal-Gonzalez I, Santini E, Quirk GJ. Consolidation of fear extinction requires NMDA receptor-dependent bursting in the ventromedial prefrontal cortex. Neuron. 2007;53:871-80.

24. Moore H, Jentsch JD, Ghajarnia M, Geyer MA, Grace AA. A neurobehavioral systems analysis of adult rats exposed to methylazoxymethanol acetate on E17: implications for the neuropathology of schizophrenia. Biol Psychiatry. 2006;60:253-64.

25. Schneider M. Adolescence as a vulnerable period to alter rodent behavior. Cell Tissue Res. 2013;354:99-106.

26. Rosenkranz JA, Grace AA. Dopamine attenuates prefrontal cortical suppression of sensory inputs to the basolateral amygdala of rats. J Neurosci. 2001;21:4090-103.

27. Zimmerman JM, Maren S. NMDA receptor antagonism in the basolateral but not central amygdala blocks the extinction of Pavlovian fear conditioning in rats. Eur J Neurosci. 2010;31:1664-70.

28. Le Pen G, Gourevitch R, Hazane F, Hoareau C, Jay TM, Krebs M-O. Peri-pubertal maturation after developmental disturbance: a model for psychosis onset in the rat. Neuroscience. 2006;143:395-405.

29. Gill KM, Miller SA, Grace AA. Impaired contextual fear-conditioning in MAM rodent model of schizophrenia. Schizophr Res. 2017;195:343-52.

30. Dieleman S, Röder CH. Emotional memory modulation in schizophrenia: an overview. Acta Psychiatr Scand. 2013;127:183-94.

31. Lodge DJ, Grace AA. Aberrant hippocampal activity underlies the dopamine dysregulation in an animal model of schizophrenia. J Neurosci. 2007; 27:11424-30.
32. Esmaeili B, Grace AA. Afferent drive of medial prefrontal cortex by hippocampus and amygdala is altered in MAM-treated rats: evidence for interneuron dysfunction. Neuropsychopharmacology. 2013;38:1871-80.

33. Laviolette SR, Lipski WJ, Grace AA. A subpopulation of neurons in the medial prefrontal cortex encodes emotional learning with burst and frequency codes through a dopamine D4 receptor-dependent basolateral amygdala input. J Neurosci. 2005;25:6066-75.

34. Gabbott PLA, Warner TA, Busby SJ. Amygdala input monosynaptically innervates parvalbumin immunoreactive local circuit neurons in rat medial prefrontal cortex. Neuroscience. 2006;139:1039-48.

35. Mcdonald AJ. Glutamate and aspartate immunoreactive neurons of the rat basolateral amygdala: colocalization of excitatory amino acids and projections to the limbic circuit. J Comp Neurol. 1996;365:367-79.

36. Shinonaga $Y$, Takada M, Mizuno N. Topographic organization of collateral projections from the basolateral amygdaloid nucleus to both the prefrontal cortex and nucleus accumbens in the rat. Neuroscience. 1994;58:389-97.

37. Lodge DJ, Behrens MM, Grace AA. A loss of parvalbumin-containing interneurons is associated with diminished oscillatory activity in an animal model of schizophrenia. J Neurosci. 2009;29:2344-54.

38. Baeg EH, Kim YB, Jang J, Kim HT, Mook-Jung I, Jung MW. Fast spiking and regular spiking neural correlates of fear conditioning in the medial prefrontal cortex of the rat. Cereb Cortex. 2001;11:441-51.

39. Perlstein WM, Carter CS, Noll DC, Cohen JD. Relation of prefrontal cortex dysfunction to working memory and symptoms in schizophrenia. Am J Psychiatry. 2001;158:1105-13.

40. Weiss AP, Heckers S. Neuroimaging of declarative memory in schizophrenia. Scand J Psychol. 2001;42:239-50.

41. McEwen BS, Morrison JH. The brain on stress: vulnerability and plasticity of the prefrontal cortex over the life course. Neuron. 2013;79:16-29.

42. Radley JJ, Arias CM, Sawchenko PE. Regional differentiation of the medial prefrontal cortex in regulating adaptive responses to acute emotional stress. J Neurosci. 2006;26:12967-76.

43. Tavares RF, Corrêa FMA, Resstel LBM. Opposite role of infralimbic and prelimbic cortex in the tachycardiac response evoked by acute restraint stress in rats. Neurosci Res. 2009;87:2601-7.

44. Gomes FV, Grace AA. Prefrontal cortex dysfunction increases susceptibility to schizophrenia-like changes induced by adolescent stress exposure. Schizophr Bull. 2017;43:592-600.

45. Rosenkranz JA, Grace AA. Cellular mechanisms of infralimbic and prelimbic prefrontal cortical inhibition and dopaminergic modulation of basolateral amygdala neurons in vivo. J Neurosci. 2002;22:324-37.

46. Quirk GJ, Beer JS. Prefrontal involvement in the regulation of emotion: convergence of rat and human studies. Curr Opin Neurobiol. 2006;16:723-7.

47. Duvarci S, Pare D. Amygdala microcircuits controlling learned fear. Neuron. 2014;82:966-80.

48. Paxinos G, Watson C. The rat brain in stereotaxic coordinates. San Diego: Academic Press; 1996.

49. Floresco SB, Tse MT. Dopaminergic regulation of inhibitory and excitatory transmission in the basolateral amygdala-prefrontal cortical pathway. J Neurosci. 2007;27:2045-57.

50. Pérez-Jaranay JM, Vives F. Electrophysiological study of the response of medial prefrontal cortex neurons to stimulation of the basolateral nucleus of the amygdala in the rat. Brain Res. 1991;564:97-101.

51. Lavin A, Moore HM, Grace AA. Prenatal Disruption of Neocortical Development Alters Prefrontal Cortical Neuron Responses to Dopamine in Adult Rats. Neuropsychopharmacology. 2005; 30:1426-1435

52. Lewis DA, Curley AA, Glausier JR, Volk DW. Cortical parvalbumin interneurons and cognitive dysfunction in schizophrenia. Trends in Neurosciences. 2012; 35:57-67 